\section{Prevalência de cárie dentária em escolares da região rural de Itapetininga, São Paulo, Brasil}

\author{
Prevalence of dental caries in schoolchildren in the \\ rural area of Itapetininga, São Paulo State, Brazil
}

Tatiana Ribeiro de Campos Mello 1 José Leopoldo Ferreira Antunes 1

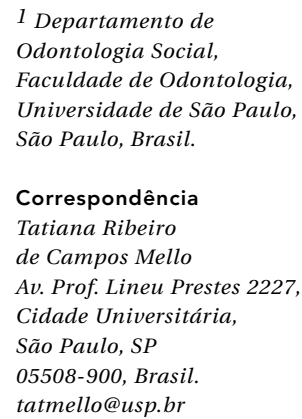

\begin{abstract}
The present study is an attempt to characterize dental needs of the rural population in Itapetininga, São Paulo State, Brazil. One single dentist examined 291 children ages 5 and 12 years in rural schools, adopting WHO criteria for oral health surveys. Parents reported their children's socioeconomic characteristics and habits. Nonprimary data gathered by the Brazilian health authority supplied information regarding the schoolchildren in the urban area of the town. Caries indices ranked as follows: $d m f t=2.63$ (5year-old children) and DMFT $=2.45$ (12-yearold children). The decayed component comprised $85.6 \%$ of the dmft and $34.2 \%$ of the DMFT, indicating limited utilization of dental treatment by children with decayed deciduous teeth. Caries prevalence was higher in rural schoolchildren than in their urban counterparts. Analysis of the results aims to improve planning of dental care.
\end{abstract}

Dental Caries; Rural Population; Dental Care for Children; Oral Health

\section{Introdução}

Nos últimos anos, o Ministério da Saúde, em nível nacional, e a Secretaria de Estado da Saúde, em São Paulo, promoveram levantamentos epidemiológicos em saúde bucal, visando fornecer dados para o planejamento de intervenções em saúde bucal. Os levantamentos mais recentes com abrangência nacional foram realizados em 1986, 1993 e 1996, e permitiram identificar uma expressiva redução da prevalência de cáries em crianças de idade escolar 1,2. Apesar disso, os estudos que analisaram os dados produzidos por esses levantamentos indicaram que, como em outros países, a melhoria global dos níveis de saúde bucal foi acompanhada pela polarização da experiência de cáries nos segmentos populacionais mais submetidos à privação ${ }^{3}$.

Na medida em que, muitas vezes, as áreas rurais brasileiras apresentam piores indicadores de renda, saneamento básico e níveis de escolaridade que as respectivas áreas urbanas 4 , acredita-se que possam configurar um importante pólo de concentração para os agravos à saúde bucal.

Também no que diz respeito à distribuição de estabelecimentos públicos e privados de saúde, sabe-se que sua maior concentração nas zonas urbanas configura outro importante fator de desigualdade no acesso aos serviços de saúde. A despeito disso, poucos estudos foram 
realizados para caracterizar as condições de saúde bucal da população rural brasileira.

"Pouco conhecimento se tem a respeito do comportamento das principais doenças bucais nos pequenos municípios brasileiros, notadamente aqueles eminentemente rurais" 5 (p. 227).

Os benefícios do desenvolvimento, tanto no Brasil como em outros países, são distribuídos de modo desigual na população, afetando, em geral, as áreas urbanas antes que as rurais, onde é maior a dificuldade em atender as necessidades de saúde da população.

Uma acentuada expansão do serviço público odontológico seguiu-se à reforma do serviço de saúde durante os anos noventa, como indicado pelo crescente número de dentistas contratados pelo Sistema Único de Saúde 6 . No entanto, o excesso de demanda por esses serviços, sua desigual distribuição geográfica e longas filas de espera ainda configuram importantes fatores de restrição ao acesso e demandam esforços suplementares de programação 7 .

Para promover a saúde bucal de um modo efetivo, a Odontologia precisa conhecer a distribuição dos agravos e das necessidades de tratamento dentário preventivo e restaurador em cada segmento de nossa sociedade, para assim prever e planejar ações de saúde específicas e adequadas às suas necessidades. Essa observação reitera a importância da utilização da Epidemiologia com o intuito de instruir o planejamento de programas e serviços de promoção de saúde bucal. Ademais, o reconhecimento das necessidades dessa população é imprescindível para adequar os cuidados de promoção em saúde bucal ao princípio da eqüidade em saúde, compatibilizando os recursos assistenciais e preventivos às efetivas necessidades da população.

O Município de Itapetininga está localizado na região Sul do Estado de São Paulo, Brasil, na bacia do Alto Paranapanema. É a terceira cidade em extensão territorial do Estado. Dados do Censo 20004 indicavam que dos seus 125.411 habitantes, 13.418 residiam em área rural.

Em 1991, o Índice de Desenvolvimento Humano (IDH) medido pelo Programa das Nações Unidas para o Desenvolvimento 8 para a cidade foi 0,778 , um pouco abaixo, portanto, do valor de referência obtido para o Estado como um todo, no mesmo período: 0,787. A esperança de vida média foi 65,1 anos de idade, enquanto o coeficiente de mortalidade infantil indicava 32,14 óbitos de menores de um ano para cada mil nascidos vivos. Apenas $34,7 \%$ das pessoas com mais de 25 anos de idade tinham mais de quatro anos de estudo e $17,2 \%$ das crianças de sete a quatorze anos não freqüenta- vam a escola em 1991. A renda familiar per capita foi em média 1,48 salários mínimos, com $26,46 \%$ das pessoas vivendo com menos de 0,5 salário mínimo.

Segundo o Sistema de Informações Ambulatoriais do Sistema Único de Saúde, em 1997, o município possuía 33 equipamentos odontológicos instalados no serviço público, sendo trinta geridos pela prefeitura, um pelo Estado e um por instituição filantrópica. A prefeitura mantinha 45 dentistas contratados para atuar nas unidades básicas de saúde, além de 27 funcionários contratados como "auxiliares odontológicos” e nenhum técnico em higiene dental. Não obstante estes serviços estarem concentrados em áreas urbanas, registrou-se a existência de cinco unidades públicas de saúde em funcionamento em áreas rurais, com seis cirurgiões dentistas e cinco auxiliares de Odontologia contratados.

O presente estudo visa mensurar a prevalência de cárie dentária em escolares de 5 e 12 residentes na área rural de Itapetininga, explorar possíveis associações com aspectos sócioeconômicos e hábitos de higiene bucal e comparar com a prevalência de cárie dentária em escolares da área urbana do município.

\section{Material e métodos}

Exames bucais foram efetuados, entre os meses de outubro e novembro de 2001, em dez estabelecimentos de ensino de primeiro grau localizados em área rural do Município de Itapetininga. Foram examinados 142 escolares de 5 anos de idade e 149 de 12 anos, correspondendo respectivamente a $67 \%$ e $55 \%$ da população de crianças de 5 e 12 anos de idade matriculadas nas escolas da região rural do município. $\mathrm{O}$ grupo estudado correspondeu à totalidade daqueles que não faltaram à escola no dia marcado para os exames e que trouxeram devidamente preenchido e assinado pelos pais ou responsáveis o termo de consentimento (cujo modelo foi previamente aprovado pelo Comitê de Ética em Pesquisa da Faculdade de Odontologia da Universidade de São Paulo - USP) distribuído previamente pelos professores com apoio das Secretarias Municipais de Educação e de Saúde, e da Delegacia de Ensino da Secretaria Estadual de Educação.

Os exames bucais foram realizados na própria escola, por uma única dentista previamente treinada e preparada pela Faculdade de Saúde Pública da USP para a aplicação dos critérios estabelecidos pela Organização Mundial da Saúde 9 em levantamentos básicos de saúde 
bucal. Os dados coletados foram registrados, com o apoio de um anotador, em fichas apropriadas para o estudo. Foram utilizados espelho bucal plano e sonda periodontal previamente esterilizados, espátula de madeira descartável e luz natural. Durante a coleta dos dados, um exame foi repetido a cada vinte observações, aproximadamente, com a finalidade de controlar a aplicação dos critérios padronizados no instrumento de aferição. Para medir a concordância intra-examinadora foi calculado o coeficiente de Kappa e o percentual de concordância global das anotações, segundo as categorias de dentes permanentes e decíduos "hígidos", “cariados", "perdidos” e "obturados".

Foi também aplicado junto aos pais e responsáveis pelos escolares um questionário que objetivava colher dados sobre as características sócio-econômicas das famílias, hábitos alimentares e de higiene dental.

As informações relativas à população urbana foram obtidas mediante apuração de dados secundários supridos pelo Levantamento Epidemiológico em Saúde Bucal 10, realizado em 1998, com o apoio da Faculdade de Saúde Pública da USP e da Secretaria de Estado da Saúde de São Paulo.

\section{Resultados}

Foram realizados 291 exames bucais nos escolares de 5 e 12 anos da área rural do município. Para medir a concordância intra-examinadora, foram reexaminados 17 escolares, obtendo-se 98,5\% de concordância global entre os exame e reexames, com índice de Kappa de 0,957.

Na Figura 1, é apresentada a distribuição dos escolares de cinco anos segundo os componentes do índice ceo-d. Pode-se observar que os escolares de áreas rurais apresentaram índice ceo-d mais elevado que os escolares de áreas urbanas. Além disso, o componente "c" (relativo à cárie não tratada) foi o que mais contribuiu para esse índice na área rural, ao contrário da área urbana, onde o componente "o" (relativo à restauração dentária) foi o mais elevado.

$\mathrm{O}$ índice CPO-D encontrado nos escolares de 12 anos e mostrado na Figura 2 foi mais elevado para os escolares da área rural. Além disso, o componente "C" foi significativamente mais elevado para essa população $(\mathrm{p}<0,05)$, indicando sua maior prevalência de cárie não tratada.

A porcentagem de escolares livres de cárie $($ ceo $=0$ ou $\mathrm{CPO}=0)$ foi maior na área urbana ao mesmo tempo que a porcentagem de escolares com alta prevalência de cárie $($ ceo $\geq 4$ ou

\section{Figura 1}

Distribuição dos escolares de 5 anos da área rural e urbana de Itapetininga, segundo os componentes do índice ceo-d.

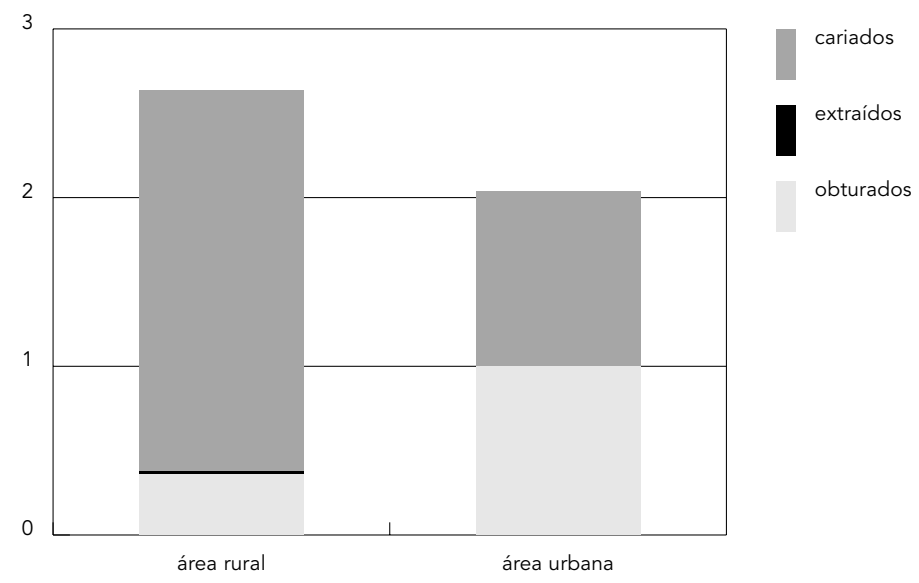

Figura 2

Distribuição dos escolares de 12 anos da área rural e urbana de Itapetininga, segundo os componentes do índice CPO-D.

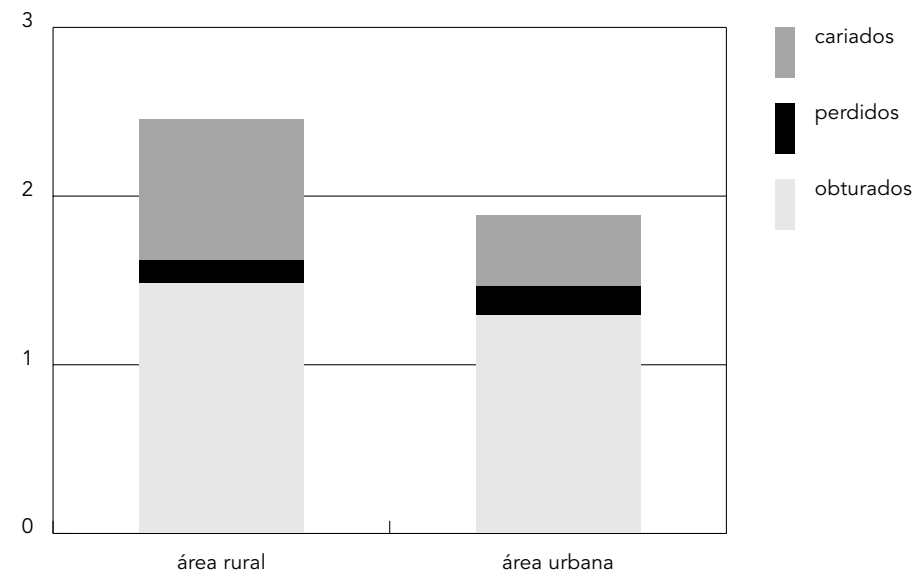

$\mathrm{CPO} \geq 4$ ) foi menor do que na área rural demonstrando que os escolares urbanos de 5 ou 12 anos possuem melhores indicativos de saúde bucal que os escolares de áreas rurais. (Tabela 1).

Na Tabela 2, são apresentados os dados de condição sócio-econômica das famílias rurais de Itapetininga. A renda pode ser considerada baixa, com $49 \%$ das famílias recebendo até um 
Número e porcentagem de escolares com 5 e 12 anos livres de cárie e com alta prevalência de cárie (4 ou mais dentes afetados), de acordo com a localização.

\begin{tabular}{|c|c|c|c|c|c|c|c|c|}
\hline \multirow[t]{3}{*}{ Condição dentária } & \multicolumn{4}{|c|}{ Rural } & \multicolumn{4}{|c|}{ Urbano } \\
\hline & \multicolumn{2}{|c|}{5 anos } & \multicolumn{2}{|c|}{12 anos } & \multicolumn{2}{|c|}{5 anos } & \multicolumn{2}{|c|}{12 anos } \\
\hline & $\mathrm{n}$ & $\%$ & $\mathrm{n}$ & $\%$ & $\mathrm{n}$ & $\%$ & $\mathrm{n}$ & $\%$ \\
\hline Livres de cárie & 55 & 39 & 53 & 36 & 39 & 46 & 51 & 52 \\
\hline \multirow[t]{2}{*}{ Alta prevalência de cárie } & 56 & 39 & 45 & 30 & 19 & 22 & 24 & 24 \\
\hline & \multicolumn{2}{|c|}{ IC95\% } & \multicolumn{2}{|c|}{ IC95\% } & \multicolumn{2}{|c|}{ IC95\% } & \multicolumn{2}{|c|}{ IC95\% } \\
\hline Livres de cárie & \multicolumn{2}{|c|}{$31 \%-47 \%$} & \multicolumn{2}{|c|}{$28 \%-44 \%$} & \multicolumn{2}{|c|}{$35 \%-57 \%$} & \multicolumn{2}{|c|}{$42 \%-62 \%$} \\
\hline Alta prevalência de cárie & \multicolumn{2}{|c|}{$31 \%-48 \%$} & \multicolumn{2}{|c|}{$23 \%-38 \%$} & \multicolumn{2}{|c|}{$14 \%-33 \%$} & \multicolumn{2}{|c|}{$17 \%-34 \%$} \\
\hline
\end{tabular}

Tabela 2

Aspectos sócio-econômicos e comportamentais das famílias residentes em áreas rurais de Itapetininga, São Paulo, Brasil.

\begin{tabular}{lrr}
\hline Aspectos sócio-econômicos & $\mathbf{n}$ & $\%$ \\
\hline Renda familiar & 134 & 49 \\
$\quad$ Até 1 salário mínimo & 140 & 51 \\
$\quad$ Mais que 1 salário mínimo & & \\
& & \\
Escolaridade do responsável & 216 & 83 \\
$\quad$ Até o 1o grau completo & 43 & 6 \\
2o grau ou superior & & \\
& & \\
Aglomerado domiciliar & 108 & 39 \\
$\quad \leq 2$ moradores por dormitório & 167 & 61 \\
> 2 moradores por dormitório & & \\
& & \\
Visitas ao CD & 147 & 54 \\
$\quad$ Nunca ou 1 vez & 125 & 46 \\
2 ou mais vezes & & \\
& & \\
Ingestão de açúcar & 247 & 90 \\
Até 1 vez ao dia & & 10 \\
2 ou mais vezes ao dia & &
\end{tabular}

salário mínimo ( $\mathrm{R} \$ 200,00)$, e $61 \%$ dessas residiam em domicílios com mais de dois moradores por dormitório. O grau de escolaridade também foi baixo: $83 \%$ dos pais ou responsáveis não estudaram ou concluíram apenas o primeiro grau. Os responsáveis declararam ainda que $90 \%$ dos escolares ingerem produtos açucarados com freqüência menor ou igual a uma vez ao dia, e que $54 \%$ nunca visitaram o cirur- gião-dentista ou fizeram isso uma vez. Esses dados de condição sócio-econômica indicaram a característica de privação social dessas famílias.

\section{Discussão}

Neste estudo foram analisados e comparados dados de cárie dentária provenientes de duas amostras populacionais de escolares, totalizando 475 observações, em áreas rural e urbana do Município de Itapetininga.

Uma primeira limitação metodológica do estudo diz respeito aos dados relativos à área urbana, os quais foram obtidos por meio de dados secundários 10 . O relatório original do levantamento não permite recuperar informações sobre concordância inter e intra-observadores para o levantamento de dados na cidade. Admite-se que possíveis vieses tenham sido minimizados no processo de treinamento e calibração dos examinadores, realizado previamente à coleta de dados.

Uma segunda limitação metodológica é tocante à elevada taxa de não-participação no estudo, para o grupo examinado na área rural. Cerca de $37 \%$ das crianças de 5 anos e $45 \%$ das crianças de 12 anos deixaram de ser examinadas por terem faltado à escola no dia programado para o levantamento ou por não terem trazido devidamente preenchido o questionário para os responsáveis, que incluía o termo de consentimento para a participação no estudo. Admite-se, no entanto, que essas perdas possam não ter reduzido o poder de inferência desses dados para o conjunto das crianças de mesma idade na região rural de Itapetininga, uma vez que o levantamento abrangeu todas as escolas localizadas nos vários distritos rurais 
da cidade. Além disso, sublinha-se o elevado valor para a estatística Kappa $(0,957)$ no reexame de escolares para a avaliação de concordância intra-examinadora, permitindo considerar como preciso o instrumento de medida.

$\mathrm{Na}$ área rural de Itapetininga, observaramse elevados indicadores de prevalência de cáries aos cinco anos de idade (Figura 1), em níveis semelhantes aos observados em localidades rurais da África por Attin et al. 11 e por Brindle et al. 12. Aos 12 anos, o CPO-D de 2,45 (Figura 2) comparava-se aos resultados de Sampaio et al. 13, pois em áreas rurais da Paraíba, onde existia uma concentração natural de flúor na água, o CPO-D aos 12 anos era de 2,5.

Em outras áreas rurais brasileiras, Hille 14 em Londrina, Marques et al. 15, em Uberlândia, e em Araraquara Dini et al. 16, observaram prevalência de cárie ainda mais elevada que a encontrada neste estudo. Em algumas comunidades rurais africanas e asiáticas, porém, foram observados melhores indicadores de saúde bucal 17,18,19,20. Entretanto, esses baixos índices foram atribuídos às características de isolamento das áreas rurais desses países, e à condição de intensa privação social dessas populações.

Os escolares residentes na área rural de Itapetininga apresentaram índices mais elevados de cáries que aqueles residentes em área urbana (Figuras 1 e 2), situação oposta à encontrada em estudos na África 20,21,22, no Iraque 19, na América Central 23 e Austrália 24. Contudo, tais regiões foram descritas pelos autores como isoladas das regiões urbanas mais próximas, em marcante contraste com a área rural de Itapetininga. Ademais, nos estudos realizados na África, deve-se considerar também a característica de intensa privação a que estavam submetidos os grupos de população estudados.

Essas observações indicam que o maior isolamento dos grupos residentes em áreas rurais, sua distância dos centros urbanos, o menor acesso a produtos industrializados açucarados, são fatores importantes para o registro de menor prevalência de cáries em populações rurais. Consistente com essa hipótese, Dini et al. 16, Jamel et al. 19, Sampaio et al. 13, Irigoyen et al. 25 e Blay et al. 22 observaram que os moradores de áreas rurais apresentavam baixo consumo de açúcar.

As famílias da área rural de Itapetininga não se encontram tão isoladas quanto o descrito nesses estudos. Além disso, pequenos distritos rurais foram criados para suprir as carências inerentes à distância da área urbana, e nesses distritos são encontrados pontos de comércio, escolas e, em alguns, o posto de saúde. Todavia, a maioria das respostas do questioná- rio sobre o consumo de açúcar indicava que esse era realizado apenas "de vez em quando". Essa indicação relativa de baixo consumo de açúcar talvez possa ser atribuída ao fato de que elevada porcentagem dessas famílias vive com um salário mínimo ou menos (Tabela 2).

De acordo com Sampaio et al. 13, no entanto, a dieta está mudando nas áreas rurais do Brasil, em função do maior acesso aos produtos industrializados e ao açúcar, o que pode provocar um aumento na prevalência de cárie dentária dessas populações. Segundo El-Nadeef et al. 26, quando as características sócio-econômicas são controladas, as diferenças na prevalência de cárie entre as áreas urbana e rural desaparecem. Essa indicação, no entanto, ainda precisa ser verificada.

Brindle et al. 12 constataram que os pais moradores em áreas rurais nunca haviam orientado os filhos quanto à importância de se visitar o cirurgião-dentista, pois naquela comunidade carente da África outros fatores eram prioritários, como a luta pela vida, já que, por causa da situação social de extrema miséria, a desnutrição infantil era alta.

A maior dificuldade de acesso a serviços de saúde bucal dos residentes em áreas rurais foi relatada em diversos estudos de áreas rurais de diferentes países 11,12,14,15,16,17,23,24,25,27,28,29,30,31,32. Essas indicações são consistentes com a presente observação de menor utilização de assistência odontológica pelas crianças de cinco anos, conforme expresso pelo percentual mais elevado do componente c (cariado) para o índice ceo-d na área rural (Figura 1).

Aos 12 anos, embora tenha sido o componente $\mathrm{O}$ (obturado) o que mais contribuiu para o índice de cárie observado, os escolares da área rural de Itapetininga possuem uma elevada porcentagem de dentes com cavidades de cárie, necessitando de tratamentos restauradores (Figura 2). Porém, a situação é ainda mais preocupante para as crianças de cinco anos, visto que o componente c (cariado) é o maior responsável pelo índice ceo-d, indicando baixa cobertura dos serviços odontológicos para essa idade (Figura 2). Situação semelhante foi verificada por Brindle et al. 12 e Sathananthan et al. 17, dado que as crianças de cinco anos desses estudos tiveram significativamente maior prevalência de cárie que as de 12 anos. Em Araraquara, Dini et al. 16 verificaram que o índice CPO-D já atingia o valor de 0,57 aos seis anos de idade, fato que sugere uma elevada experiência de cárie, em virtude do pequeno número de dentes permanentes erupcionados nessa idade.

No presente estudo, a baixa incorporação de tratamento odontológico restaurador nas 
crianças de cinco anos de idade é indicativo da falta de conhecimento dos pais sobre a importância da dentição decídua. Essa observação pode também estar refletindo o fato de que os programas de saúde bucal promovidos pelo serviço público odontológico estavam única ou preferencialmente atendendo as crianças de seis anos ou mais, como pôde ser observado durante visita realizada nos postos de saúde instalados na área rural do município. Nesse sentido, sublinha-se a importância de um serviço eqüitativo de saúde bucal atentar para as necessidades de diferentes grupos etários da população.

Semelhante à situação notada em Araraquara por Dini et al. 16, na área rural de Itapetininga, ainda que a maioria dos responsáveis tenha respondido que seus filhos escovam os dentes 2 ou 3 vezes por dia, o exame bucal realizado permitiu constatar serem precárias as condições de higiene oral, sugerindo que essa resposta possa não ser confiável. Essa observação pode também estar refletindo deficiências no processo de escovação efetuado pelas crianças, pois $61 \%$ dos responsáveis pelas crianças de cinco anos da área rural de Itapetininga responderam que elas escovam seus dentes sozinhas, sem apoio ou supervisão dos adultos.

Marques et al. 15 concluíram que a alta prevalência de cárie encontrada na área rural de

\section{Resumo}

O presente estudo visa mensurar a prevalência de cárie dentária em escolares residentes na área rural de Itapetininga, São Paulo, Brasil. Uma única dentista efetuou o exame bucal de 291 crianças de 5 e 12 anos em escolas rurais, seguindo os critérios da Organização Mundial da Saúde. Características sócio-econômicas e hábitos das crianças foram informados pelos pais ou responsáveis. Dados secundários relativos aos escolares da área urbana foram utilizados para análise comparativa. Os índices de cárie observados foram: ceo-d (5 anos) de 2,63 e CPO-D (12 anos) de 2,45. O componente cariado correspondeu a $85,6 \%$ do ceo-d e $34,2 \%$ do $C P O-D$, indicando a menor utilização de serviços odontológicos pelas crianças com cáries na dentição decídua. A prevalência de cárie foi mais elevada na área rural do que na área urbana de Itapetininga. O presente estudo apresenta informações epidemiológicas inéditas para o município, oferecendo subsídios para o planejamento estratégico e normativo das ações de saúde bucal no sistema local de saúde.

Cárie Dentária; População Rural; Assistência Odontológica para Crianças; Saúde Bucal
Uberlândia se devia à desinformação sobre saúde bucal, dificuldade de acesso ao tratamento odontológico e a fatores econômicos, condições comuns a grupos de população rural de diferentes regiões brasileiras.

\section{Conclusões}

Os estudos estimando a prevalência das principais doenças bucais na população rural brasileira são escassos, o que dificulta o planejamento da intervenção em saúde bucal. A inexistência de informação anterior sobre essa condição na população infantil em Itapetininga impede a avaliação de tendências na distribuição dos agravos.

Os escolares da área rural de Itapetininga apresentaram maior prevalência de cáries e menor incorporação de serviços odontológicos do que os escolares residentes na área urbana do município; além disso, apresentaram indicações de privação social. O presente estudo apresenta informações epidemiológicas inéditas para o município, oferecendo subsídios para o planejamento estratégico e normativo das ações de saúde bucal no sistema local de saúde, com o intuito de contribuir para a eqüidade em saúde.

\section{Colaboradores}

T. R. C. Mello concebeu e planejou o estudo; aplicou os questionário e realizou os exames bucais; analisou e interpretou os resultados; redigiu e revisou o texto. J. L. F. Antunes orientou o levantamento de dados e contribuiu no planejamento do estudo, na análise e interpretação dos resultados, na redação e revisão do texto.

\section{Referências}

1. Narvai PC, Frazão P, Castellanos RA. Declínio na experiência de cárie em dentes permanentes de escolares brasileiros no final do século XX. Odontologia e Sociedade 1999; 1:25-9.

2. Narvai PC, Castellanos RA, Frazão P. Prevalência de cárie em dentes permanentes de escolares do Município de São Paulo, SP, 1970-1996. Rev Saúde Pública 2000; 34:196-200.

3. Antunes JLF, Frazão P, Narvai PC, Bispo CM, Pegoretti T. Spatial analysis to identify differentials in dental needs by area-based measures. Community Dent Oral Epidemiol 2002; 30:133-42. 
4. Fundação Instituto Brasileiro de Geografia e Estatística. Recenseamento geral da população: 2000. http://www.ibge.gov.br (acessado em 02/Ago/2002).

5. Furtado A, Traebert JL, Marcenes WS. Prevalência de doenças bucais e necessidade de tratamento em Capão Alto, Santa Catarina. Rev ABO Nacional 1999; 7:226-30.

6. Junqueira SR. Financiamento da saúde, indicadores sociais e recursos odontológicos dos municípios do Estado de São Paulo no final do século XX [Dissertação de Mestrado]. São Paulo: Faculdade de Odontologia, Universidade de São Paulo; 2001.

7. Botazzo C. Unidade básica de saúde: a porta do sistema revisitada. Bauru: Edusc; 1999.

8. Programa das Nações Unidas para o Desenvolvimento/Instituto de Pesquisa Econômica Aplicada/Fundação João Pinheiro/Fundação Instituto Brasileiro de Geografia e Estatística, 1998. Atlas do Desenvolvimento Humano no Brasil. Brasília: Programa das Nações Unidas para o Desenvolvimento.

9. World Health Organization. Oral health surveys: basic methods. 4th Ed. Geneva: World Health Organization; 1997.

10. Núcleo de Estudos e Pesquisas de Sistemas de Saúde, Faculdade de Saúde Pública, Universidade de São Paulo. Levantamento Epidemiológico em Saúde Bucal: Estado de São Paulo, 1998. São Paulo: Faculdade de Saúde Pública, Universidade de São Paulo; 1999.

11. Attin T, Mbiydzemo FN, Villard I, Kielbassa AM, Kielbassa AM, Hellwig E. Dental status of schoolchildren from a rural community in Cameroon. South African Dental Journal 1999; 54:145-8.

12. Brindle R, Wilkinson D, Harrison A, Connolly C, Cleaton-Jones P. Oral heath in Hlabisa, KwaZulu/ Natal - a rural school and community based survey. Int Dent J 2000; 50:13-20.

13. Sampaio FC, Hossain NA, von der Fehr FR, Ameberg P. Dental caries and sugar intake of children from rural areas with different water fluoride level in Paraíba, Brazil. Community Dent Oral Epidemiol 2000; 28:307-13.

14. Hille AL. Contribuição para modelo de plano de saúde dental em área rural concentrada [Tese de Doutorado]. Londrina: Universidade Estadual de Londrina; 1976.

15. Marques DE, Rink MCM, Loureiro RMT, Silva VC. Levantamento epidemiológico de cárie dentária na zona rural de Uberlândia, Minas Gerais; contribuição para um modelo de programa de saúde bucal. Revista do Centro de Ciências Biomédicas da Universidade Federal de Uberlândia 1986; 2:33-8.

16. Dini EL, Vertuan V, Pincelli CAS. Condições bucais de escolares da área rural do Município de Araraquara-SP. Revista de Odontologia da UNESP 1993; 22:125-33.

17. Sathananthan K, Vos T, Bango G. Dental caries, fluoride levels and oral hygiene practices of school children in Matebeland South, Zimbabwe. Community Dent Oral Epidemiol 1996; 24:21-4.

18. Milsom KM, Rijal K, Lennon MA. Oral health status of 12-year-old children in Nepal in 1994. Int Dent J 1997; 47:88-93.
19. Jamel HA, Sheiham A, Watt RG, Cowell CR. Sweet preference, consumption of sweet tea and dental caries; studies in urban and rural Iraqi populations. Int Dent J 1997; 47:213-7.

20. Petersen PE, Mzee MO. Oral health profile of schoolchildren, mothers and schoolteachers in Zanzibar. Community Dent Health 1998; 15:265-2.

21. Moalic É, Zérilli A, Capo-Chichi S, Apovi G. Oral and dental health of a population of school children from the Zou region of Benin (1998). Sante 1999; 9:273-6.

22. Blay D, Astrom NA, Haugejorden O. Oral hygiene and sugar consumption among urban and rural adolescents in Ghana. Community Dent Oral Epidemiol 2000; 28:443-50.

23. Alonge OK, Naredran S. Dental caries experience among school children in St. Vincent and The Grenadines: report of the first national oral health survey. Community Dent Health 1999; 16:45-9.

24. Brennan DS, Spencer AJ, Slade GD. Caries experience among publicly-funded dental patients in Australia, 1995-96: Type of care and geographic location. Aust Dent J 2000; 45:37-45.

25. Irigoyen ME, Luengas IF, Yashine A, Mejia AM, Maupome G. Dental caries experience in Mexican schoolchildren from rural and urban communities. Int Dent J 2000; 50:41-5.

26. El-Nadeef MA, Adegbembo AO, Honkala E. The association of urbanisation with the prevalence of dental caries among schoolchildren in Nigeria new capital territory. Int Dent J 1998; 48:44-9.

27. Canton L, Doño R, Bellagamba S, Schmunis S. Atención primária en una comunidad rural. Informe preliminar. Revista de la Faculdad de Odontología de Buenos Aires 1985; 5:15-22.

28. Manji F, Baelum V, Fejerskov O. Tooth mortality in an adult rural population in Kenya. J Dent Res 1988; 67:496-500.

29. Ntabaye MK, Scheutz F, Poulsen S. Patient satisfaction with emergency oral health care in rural Tanzania. Community Dent Oral Epidemiol 1998; 26:289-95.

30. Crowley SJ, Campain AC, Morgan MV. An economic evaluation of a publicly funded dental prevention programme in regional and rural Victoria: an extrapolates analysis. Community Dent Health 2000; 17:145-51.

31. Mattila ML, Rautava P, Sillanpa M, Paunio P. Caries in five-year-old children and associations with family-related factors. J Dent Res 2000; 79:875-81.

32. Teixeira ACS, Rocha PLOM, Zanetti CHG. A zona rural de Planaltina e a proposta de um modelo viável de atenção em saúde bucal. Curso de Especialização em Saúde Coletiva - Faculdade de Ciências da Saúde, Universidade de Brasília. http: //www.saudebucalcoletiva.unb.br (acessado em 26/Mai/2001).

Recebido em 01/Set/2003

Versão final reapresentada em $02 / \mathrm{Fev} / 2004$

Aprovado em 03/Fev/2004 